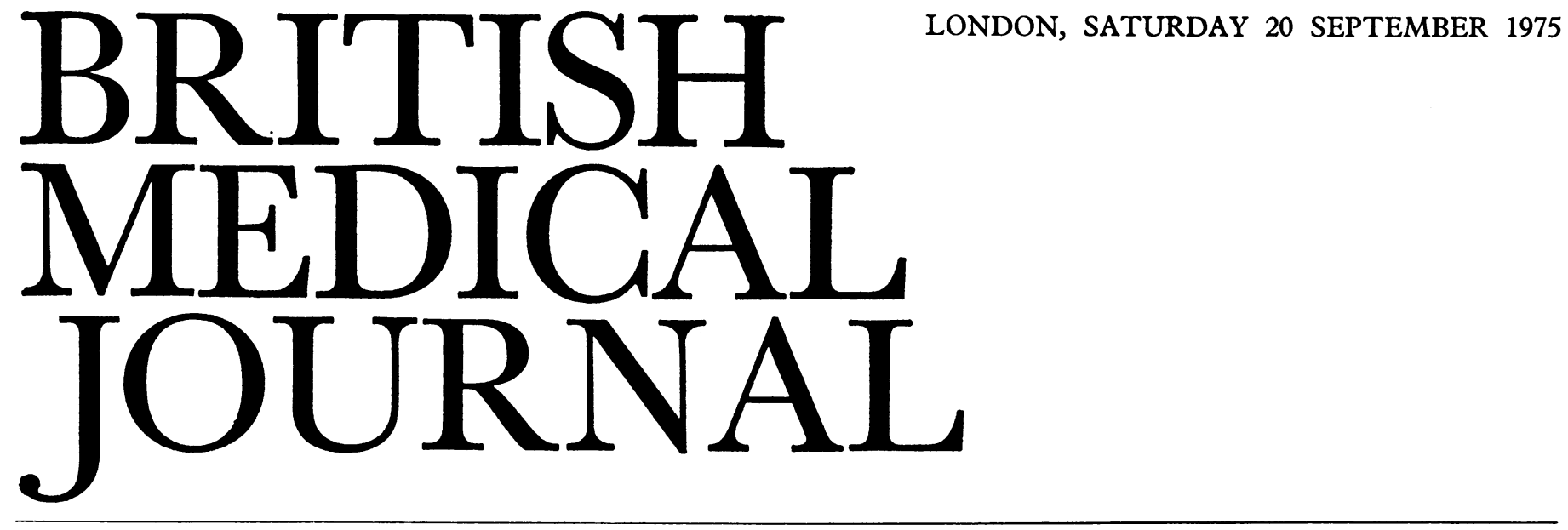

Scoliosis remains, as it has for many years, the most difficult of the problems facing the orthopaedic surgeon. Over the last decade it has been the subject of much valuable research, concerned with both advances in our understanding of the cause and new and powerful methods of correction, but many problems remain unsolved.

Although so called idiopathic scoliosis is the most common form (in an Edinburgh survey occurring in 4:1000 girls) it is only one of many types of scoliosis; indeed, at least 50 diseases are known to cause spinal curvature. Most frequent, next to idiopathic scoliosis, is congenital scoliosis due to congenital vertebral anomalies. Scoliosis is common also in many of the inherited myopathies and neuropathies, and it may often be seen as a manifestation of the very numerous but individually rare inherited diseases. Thought must always be given to the underlying cause when a structural scoliosis is investigateddetailed examination and investigation are essential in every new case, for with such a huge array of possible aetiologies it is only the alert observer who will not make mistakes.

While it is by far the most common the aetiology of idiopathic scoliosis remains unexplained. Nevertheless, the pioneer work of Wynne-Davies ${ }^{1}$ of Edinburgh and of MacEwen and Cowell ${ }^{2}$ of Wilmington has shown that a familial basis is present. Indeed, there is now a tendency in the United States to relabel idiopathic scoliosis "genetic scoliosis," but this is going ahead of the facts. What has been shown in the Edinburgh series is that in the families of the 200 children with idiopathic scoliosis investigated there was about 30 times the expected frequency of scoliosis among their first degree relatives. Indeed, when the mothers and fathers were $x$-rayed to detect small curvestoo small to pick up clinically-the number of curves over $10^{\circ}$ among the parents rose to 179 per 1000 . Occasional families show almost a dominant pattern of inheritance, but commonly it is much more a multifactorial pattern. Genetically, idiopathic scoliosis may prove to be more than one entity. After so much effort and so much time we have perhaps the first clues to a possible cause of this common problem but still have no idea of its pathogenesis, the mechanics of curve development. Robin and Stein ${ }^{3}$ have recently recorded yet one more failure to find an animal model by which to elucidate this.

Severe, usually neglected curves require forcible correction before fusion. Harrington ${ }^{4}$ was the first to develop a generally successful method of instrumentation, and this is still the best and safest of all the methods. Later Dwyer ${ }^{5}$ developed the anterior thoracoabdominal approach to the spine with anterior vertebral osteotomy followed by cabling. Almost at the same time Dewald ${ }^{6}$ and more recently $\mathrm{O}^{\prime}$ Brien and $\mathrm{Yau}^{7}$ have described the use of the halo-pelvic apparatus, in which pelvis and cranium are fixed by rods and screws to circular hoops and the spine distracted by elongation of vertical bars between the hoops. These methods have now been used for long enough for it to become apparent that with such powerful correction the spinal cord and peripheral nerves are at risk. Both MacEwen $^{8}$ and Ransford and Manning ${ }^{9}$ have described these complications, of which paraplegia is the most common as well as the most serious. Despite these problems all three methods, particularly Harrington rods, have a valuable place in the surgeon's repertoire when properly managed.

Much safer than instrumentation is plaster correction followed by fusion, but it is most suitable only in early cases. Why is it that even now it is common to see late cases requiring potentially dangerous instrumental salvage operations rather than the early, easily treated curvature? We have known for years ${ }^{10-12}$ how to determine from the earliest stages which curves will be serious, but even orthopaedic surgeons do this less often than is desirable. Everyone-general practitioners, paediatricians, and physicians-must be alerted to the serious outcome of almost any structural scoliosis; too often nothing is done and the patient reassured. It must be reiterated that early treatment, whether by Milwaukee brace or plaster correction and fusion, does control scoliosis and these children can be returned to an almost normal state if treated early enough, like so much else in children's deformities. The present enthusiasm for instrumentation is because late cases continue to present. Ideally such methods should rarely be necessary.

Does scoliosis matter? Apart from the ugliness, compression of the heart and lungs leads to a premature death for many affected patients. Nachemson ${ }^{13}$ and Nilsonne ${ }^{14}$ showed that in untreated idiopathic scoliosis nearly one-third of the patients were permanently disabled, another third were dyspnoeic walking, and none could do hard work; $76 \%$ of the girls were not married; and mortality was twice that expected for the age group, almost entirely from respiratory disease, the consequence of the scoliosis.

${ }^{1}$ Wynne-Davies, R., Fournal of Bone and foint Surgery, 1968, 50B, 24.

MacEwen, G. D., and Cowell, H. R., Fournal of Bone and foint Surgery, 1970, 52A, 405.

${ }^{3}$ Robin, G. C., and Stein, H., Journal of Bone and Foint Surgery, 1975, $57 \mathrm{~B}, 142$.

${ }^{4}$ Harrington, P. R., fournal of Bone and foint Surgery, 1962, 44A, 591. 
5 Dwyer, A. F., Newton, N. C., and Sherwood, A. A., Clinical Orthopaedics and Related Research, 1969, 62, 192.

${ }^{6}$ Dewald, R. L., and Ray, R. D., Fournal of Bone and Foint Surgery, 1970, 52A, 233.

' O'Brien, J. P., et al., fournal of Bone and foint Surgery, 1971, 53B, 217.

${ }^{8}$ MacEwen, G. D., Bunnell, W. P., and Sriram, K., fournal of Bone and foint Surgery, 1975, 57A, 404.

${ }^{9}$ Ransford, A. O., and Manning, C. W. S. F., fournal of Bone and foint Surgery, 1975, 57B, 131.

10 Ponseti, I. V., and Friedman, B., Fournal of Bone and foint Surgery, 1950, 32A, 381.

11 James, J. I. P., Fournal of Bone and foint Surgery, 1951, 33B, 399.

12 James, J. I. P., Scoliosis. E. \&. S. Livingstone, Edinburgh \& London, 1967.

13 Nachemson, A., Acta Orthopaedica Scandinavica, 1968, 39, 466.

14 Nilsonne, U., and Lundgren, K-D., Acta Orthopaedica Scandinavica, $1968,39,456$.

\section{Reye's Syndrome}

A clinician presented with a child, previously well, who has developed convulsions or disturbances of consciousness or both proceeding rapidly to coma is faced with a bewildering range of differential diagnoses. Head injuries, vascular accidents, intracranial space-occupying lesions, and direct infection of the central nervous system must be excluded on the basis of the clinical features and by appropriate investigations. Exogenous toxins may need to be looked for as well as metabolic disorders such as hypernatraemia, hypoglycaemia, diabetic acidosis, hypocalcaemia, hypomagnesaemia, uraemia, water intoxication, and acute porphyria. Hypertensive encephalopathy occurring early in glomerulonephritis before a renal lesion is suspected may also cause confusion. Also to be considered is acute toxic encephalopathy, a poorly understood condition in which convulsions and coma with fever and vomiting develop during such illness as upper respiratory tract infections, gastroenteritis, dysentery, pneumonia, or one of the exanthemata. Only supportive and symptomatic treatment is available for this condition.

With such a range of differential diagnoses, it is disappointing but understandable that another well-recognized cause of coma and convulsions in this age group, Reye's syndrome or encephalopathy with fatty degeneration of the viscera, is often not recognized in life $^{1} 12$ years after Reye, Morgan, and $\mathrm{Baral}^{2}$ first drew attention to it. Classically there may be a history of a mild prodromal illness from which the child is apparently recovering. ${ }^{3-5}$ Vomiting, which may be severe, may precede central nervous system symptoms by 3-72 hours, but it does not occur in all cases. The common course is rapid progressive deterioration of consciousness, with convulsions proceeding to coma and decerebrate posturing, death often following in 24-48 hours. There are no focal central nervous system signs, nor meningismus. Papilloedema is unusual early in the course. Hyperpnoea or irregular respiration should arouse diagnostic suspicion. Mild to moderate hepatomegaly may indicate visceral lesions, but it is absent in more than half of cases. Jaundice is unusual.

Only by doing liver function tests is the diagnosis likely to be suspected in life. If two of the following criteria are satisfied without any other obvious explanation for the clinical and biochemical features, Reye's syndrome is a probable diagnosis: aspartate aminotransferase level more than two and a half times normal, a prothrombin activity of less than $60 \%$ of normal, and a blood sugar of less than $3 \mathrm{mmol} 1 \mathrm{or}$ a C.S.F. glucose of less than $2 \mathrm{mmol} 1$. A blood ammonia level higher than $0.1 \mu \mathrm{mol} / 1$ further supports the diagnosis, ${ }^{6}$ but hyperammonaemia may be transient. Raised serum levels of alanine, lysine, and glutamine and a low citrulline are the typical amino-acid pattern. ${ }^{7}$ Confirmation of the diagnosis requires liver biopsy, but this is frequently ruled out by the prolonged prothrombin time. There is variable but often intense fatty infiltration of the liver, with diffuse vacuolation of the hepatocytes without nuclear displacement and no hepatocellular necrosis. ${ }^{8}$

Since the diagnosis of Reye's syndrome is difficult its incidence may be higher than is generally realized. About $40 \%$ of children diagnosed in life die. ${ }^{9}$ Features associated with poor prognosis include a blood ammonia level higher than $200 \mu \mathrm{mol}$ l, a rapid progression to deep coma, a prothrombin time prolonged more than twice normal, increased intracranial pressure, ${ }^{10}$ and electroencephalographic abnormalities. ${ }^{11}$

The aetiology and pathophysiology remain poorly understood. Some evidence links a variety of this syndrome in Thailand with aflatoxin ingestion. ${ }^{12} \mathrm{~A}$ viral aetiology has been suggested owing to the recovery of viruses from the nasopharynx and faeces in individual cases and on epidemiological grounds, ${ }^{13}$ but there is no direct viral invasion of liver or brain. The apparent concentration of 13 cases in one part of Canada which had been sprayed heavily with insecticide was followed by an experimental study in mice, in which pathological features similar to those in Reye's syndrome were produced by a virus in those previously exposed to insecticides. Neither the insecticides individually or in combination nor the virus caused similar pathological features. ${ }^{14}$ Even the cause of the encephalopathy remains undetermined.

For the time being the treatment of Reye's syndrome remains supportive and empirical-namely, the correction of hypoglycaemia, electrolyte abnormalities, acidosis, and hypoxia. Artificial ventilation may be required. A reduced fluid intake-10\% dextrose with maintenance electrolytes-is advised initially to minimize the risk of cerebral oedema, but this may have to be modified if the patient is dehydrated or if inappropriate release of antidiuretic hormone occurs. Neomycin by nasogastric tube and laxatives and enemata to minimize ammonia reabsorption from the gut seem rational treatment, but they are not of proved value, ${ }^{17}$ nor is dexamethasone or mannitol, the usual treatment for increased intracranial pressure. ${ }^{10}$ The bleeding diathesis may require correction with fresh frozen plasma or whole blood. The apparent beneficial effects of peritoneal dialysis ${ }^{18}$ and exchange transfusion ${ }^{17}$ have not been confirmed in some subsequent studies. ${ }^{1019}$ Potentially hazardous recommendations that have been associated with recovery include glucose and insulin, ${ }^{20}$ L-citrulline, ${ }^{1516}{ }^{21}$ and nicotinic acid. ${ }^{22}$ All must be considered of unproved value. No data are available on optimum calorie intake nor on how they should be provided.

The acute nature of this syndrome, its sporadic occurrence, and its variable severity caused difficulties in assessing the value of therapeutic regimens as well as in sorting out the pathophysiology, a necessary requisite for rational therapy. Co-operative studies may resolve these difficulties, but they will be successful only if Reye's syndrome is considered during life in all children with acute encephalopathy of unknown cause.

${ }^{1}$ Kearney, P. J., Deasy, P. F., and O'Donohoe, N. V., fournal of the Irish Medical Association, 1975, 68, 169.

Reye, R. D. K., Morgan, G., and Baral, J., Lancet, 1963, 2, 749.

3 British Medical fournal, 1973, 3, 308.

4 Lancet, 1974, 2, 445.

${ }^{5}$ Mowat, A. P.. Archives of Disease in Childhood, 1973, 48, 411

${ }^{6}$ Glasgow, A. M., Cotton, R. B., and Dhiensiri, K., American fournal of Diseases of Children, 1972, 124, 827.

' Hilty, M. D., Romshe, C., and Delamater, P. V., fournal of Pediatrics, $1974,84,362$. 